\title{
Psychische und Verhaltensstörung durch Kokain, Amphetamine, Ecstasy und Halluzinogene
}

\author{
Alexander Glahn, Thomas Hillemacher, Stefan Bleich
}

In bestimmten Bevölkerungsgruppen ist der Konsum psychotroper Substanzen geläufig und führt auch häufig zur Abhängigkeit. Neben der Pharmakotherapie zur Akutbehandlung sind langfristig Verhaltenstherapie und sozialpsychiatrische Maßnahmen erfolgreich.

\section{Allgemeine Grundlagen und Abhängigkeitskriterien}

Eine Drogenabhängigkeit ist eine psychische Erkrankung mit oftmals schwerwiegenden Veränderungen der Lebensqualität. Betroffene können darüber hinaus langfristig aus sozialen Strukturen unserer Gesellschaft herausfallen. Abhängigkeitserkrankungen sind in der Regel Folge von psychischen Lernprozessen sowie neurobiologischen Veränderungen des Gehirns [1, 2]. Das Wissen über Menge und Häufigkeit des Drogenkonsums reicht für die Diagnosestellung einer Abhängigkeitserkrankung nicht aus. Der Konsum abhängigkeitserzeugender Substanzen ist jedenfalls nicht sofort mit dem Vorliegen einer Abhängigkeitserkrankung gleichzusetzen.

Generell wird zwischen einer Abhängigkeit und schädlichem Gebrauch unterschieden.

Abhängigkeit. Die Kriterien einer Abhängigkeit sind jeweils in den gängigen Klassifikationssystemen (ICD10 und DSM-5 festgelegt) $[3,4]$.

Schädlicher Gebrauch. Ein schädlicher Gebrauch wird gemäß ICD-10 definiert als ein Konsum psychotroper Substanzen, der zu einer Gesundheitsschädigung führt. Diese kann psychischer (z.B. als depressive Episode) aber auch körperlicher Natur (z. B. Infektionskrankheiten nach Selbstinjektion der Substanz) sein. Die Unter-
ICD-10 Kriterien der Abhängigkeits-

\section{erkrankung [3]}

Zur Diagnose eines Abhängigkeitssyndroms müssen nach der ICD-10 mindestens 3 der folgenden Kriterien während des letzten Jahres gemeinsam erfüllt gewesen sein:

- starkes, oft unüberwindbares Verlangen, die Substanz einzunehmen

- Schwierigkeiten, die Einnahme zu kontrollieren (was den Beginn, die Beendigung und die Menge des Konsums betrifft)

- körperliche Entzugssymptome

- Benötigen immer größerer Mengen, damit die gewünschte Wirkung eintritt

- fortschreitende Vernachlässigung anderer Verpflichtungen, Aktivitäten, Vergnügen oder Interessen (das Verlangen nach der Droge wird zum Lebensmittelpunkt)

- fortdauernder Gebrauch der Substanz(en) wider besseres Wissen und trotz eintretender schädlicher Folgen

scheidung und Einteilungen in Abhängigkeit (Substance Dependence) beziehungsweise einem schädlichen Gebrauch einer Substanz (Substance Abuse) besteht im DSM-5 nicht mehr und wurde durch den Begriff der Substanzgebrauchsstörung (Substance Use Disorder) ersetzt [4]. 


\section{Epidemiologie}

Gemäß der aktuellen Studienlage variieren im Gegensatz z. B. zur Alkoholabhängigkeit epidemiologische Angaben bzgl. der verschiedenen Substanzen, was insbesondere zahlreicher methodischer Probleme (Illegalität von Drogen, Vertrieb) geschuldet ist [2].

Charakteristisch ist der häufig polyvalente Konsum und schädliche Gebrauch mehrerer Substanzen.

Ein episodischer Konsum tritt häufig auf. In den meisten Fällen sind spezifische Szenegruppen im Freizeitund Berufsbereich, aber auch soziale Randgruppen betroffen. Initial werden diese Substanzen häufig zur Leistungssteigerung und Erweiterung des individuellen Erlebnis- und Erfahrungsbereichs genommen. Suchtentwicklungen kommen außer bei Halluzinogenen relativ häufig vor, wobei repräsentative Daten aufgrund o.g. Problematik rar sind.

Erfahrungen mit Kokain haben 2\% der 12- bis 25-Jährigen. In definierten Altersgruppen (junge Erwachsene, in Großstädten) ist die Lebenszeitprävalenz für Kokain in den letzten Jahren angestiegen (bis auf 5-10\%) [5]. Im Verlauf der Kokainabhängigkeit zeigt sich eine erhebliche Tendenz zur Dosissteigerung. Ein Teil der erwachsenen Kokainabhängigen reduziert den Konsum nach etwa 10 Jahren oder stellt ihn insgesamt ein [1].

Erfahrungen mit Amphetaminstimulanzien (Amphetamin und Methamphetamin [Speed]) haben ca. 3,5\% der Erwachsenen und etwa 6\% der 18 - bis 29-Jährigen, wobei der Altersgipfel bei 20 - 24 Jahren liegt. Männer sind insgesamt häufiger betroffen als Frauen (etwa 3:1) $[1,6,7]$. Ecstasy-Erfahrungen weisen $0,5-4 \%$ der Erwachsenen und bis zu 5\% der Jugendlichen in Europa auf. Der Altersgipfel liegt bei 12 - 25 Jahren [7]. Lysergsäurediethylamid (LSD) wird am häufigsten in der Gruppe 18- bis 20-Jähriger konsumiert, seit Anfang der 1990er-Jahre spielt LSD bei den Jugendlichen aber eine eher untergeordnete Rolle, deutlich verbreiteter sind psilocybinhaltige Pilze. Hier weisen mehr als 6\% der 21- bis 24-Jährigen Konsumerfahrungen auf [6].

\section{Ätiopathogenese}

Bestimmte Risikofaktoren können das Auftreten von Abhängigkeitserkrankungen erhöhen. Hierzu gehören:

- das Vorliegen von Abhängigkeitserkrankungen bei leiblichen Eltern und Großeltern
- eine mangelhafte Impulskontrolle

- ein vermindertes Vorhandensein von Problembewältigungsstrategien

- die Prägung im Umgang mit Suchtstoffen durch das soziale Umfeld [2]

\section{Symptomatik}

Die einzelnen Substanzklassen führen zu einer spezifischen Symptomatik, auf die im Verlauf dieses Artikels noch weiter eingegangen wird, sodass immer zwischen einer substanzspezifischen Symptomatik, z. B. während einer Intoxikation oder eines Entzugs, und den unspezifischen Symptomen einer Abhängigkeitserkrankung zu unterscheiden ist [2]. Eine allgemeingültige Aussage zum Verlauf und zur Prognose bei Abhängigkeitserkrankungen ist nicht möglich, da die Prognose von verschiedenen Faktoren abhängig ist (abhängigkeitserzeugende Potenz, Art des Konsums, individuelle Disposition etc.).

\section{Diagnose und Differenzialdiagnose}

Die Psychodiagnostik dient der Abklärung substanzbedingter Störungen nach dem Diagnoseschlüssel des ICD-10 (Intoxikation, schädlicher Gebrauch, Abhängigkeit, Entzugssyndrom, substanzinduzierte Störungen). Neben der ausführlichen Suchtanamnese sind der psychopathologische Befund, die Behandlungsmotivation und komorbide Störungen zu erheben.

Eine ausführliche Somato- und Sozialdiagnostik ist obligat.

Ergänzend können standardisierte und halbstandardisierte Interviews herangezogen werden, wobei das CIDI (Composite International Diagnostic Interview) mit dem substanzspezifischen Frageteil CIDI-SAM (Substance Abuse Module) direkt zur Diagnosestellung nach ICD-10 verwendet werden kann [8, 9].

\section{Therapie}

Die Therapieziele der verschiedenen Drogenabhängigkeiten variieren im Einzelfall. Suchtmittelfreiheit ist zwar wünschenswert und sollte oberstes Ziel der Behandlung sein, ist aber nicht bei allen Patienten zu erreichen. Vorrangige Ziele der Psychotherapie innerhalb der Akutbehandlung sind: 
- Festigung der Behandlungsmotivation

- die Vermittlung positiver Erfahrungen im Rahmen einer Psychotherapie

- die Ausarbeitung individueller Behandlungsziele, die in der Phase der Postakutbehandlung bzw. der Rehabilitation aufgegriffen werden können [1,2]

Betroffene zeigen zu Beginn häufig wenig Krankheitseinsicht oder bagatellisieren ihr Suchtproblem, sodass eine wichtige Herausforderung in der Kontaktphase das Schaffen von Veränderungsmotivation ist. Hier ist insbesondere die motivierende Gesprächsführung geeignet [10].

\section{Merkmale der motivierenden}

\section{Gesprächsführung [10]}

- empathische Grundhaltung mit Verzicht auf Konfrontation

- Förderung der Wahrnehmung von Diskrepanzen zwischen Selbstbild und Wirklichkeit

- Aufbau von Selbstvertrauen bezüglich der Fähigkeit zur Abstinenz

- Vereinbarung von gemeinsam erarbeiteten und umschriebenen Behandlungszielen

An Techniken kommen u. a. offene Fragen ohne implizite Wertung, reflektierendes Zuhören, positive Rückmeldungen und eine strukturierte Zusammenfassung der Äußerungen des Patienten zur Anwendung.

\section{Substanzspezifische Informationen}

\section{Kokain}

Kokain ist ein Extrakt aus den Blättern des Kokastrauches, kann nasal aspiriert (Abb.1), geraucht, injiziert oder direkt auf die Schleimhäute aufgetragen werden. Die intravenöse Applikation hat eine Bioverfügbarkeit von $100 \%$, wohingegen beim Inhalieren (Rauchen) oder Schnupfen die Bioverfügbarkeit bei ca. 25\% liegt. Die Halbwertszeit im Plasma liegt zwischen 40 und $60 \mathrm{~min}$ und der Metabolismus erfolgt in Leber und Plasma [11]. Der Hauptmetabolit lässt sich im Urin etwa bis zu 3 Tage nachweisen, bei chronischem Konsum mit bis zu 15-22 Tagen deutlich länger [2].

Kokain ist ein sehr wirksames Lokalanästhetikum, führt zu einer Verengung der Blutgefäße und besitzt starke psychotrope Effekte. Neurochemisch hemmt

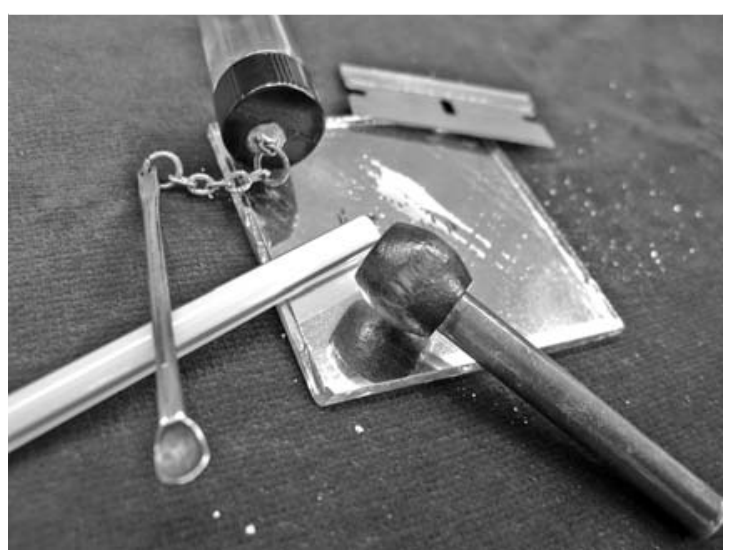

Abb. 1 Oben ein Glasgefäß für Kokainpulver mit Schraubverschluss, an dem ein Portionierungslöffelchen hängt. Auf der Spiegelfläche eine „Line“. Darunter, zum Inhalieren: ein Strohhalm und ein Röhrchen mit Holznuss, die das Nasenloch beim Inhalieren abdichtet (Quelle: Thieme Verlagsgruppe).

Kokain dabei die Wiederaufnahme von Dopamin, Serotonin und Noradrenalin über eine Blockade des Dopamintransporters. Entscheidend für die Erzeugung von Abhängigkeit scheinen die dopaminergen Fasern im Mittelhirn zu sein, die das ventrale Striatum, aber auch andere striatale Zellen versorgen. Eine Hemmung der Wiederaufnahme von Dopamin im mesolimbischen Dopaminsystem führt zu einer erhöhten Kon-

\section{Sonderform: Crack}

Crack ist eine mit Backpulver vermischte Aufbereitung des Kokains und eine der am stärksten abhängigkeitserzeugenden Drogenmischungen. In den USA konsumierten 2007 1,5 Mio. Personen Crack. Am häufigsten wird Crack von 18- bis 25-jährigen Personen konsumiert [2].

zentration von Dopamin im synaptischen Spalt [12].

Klinische Symptome. Kokain ist ein starkes Psychostimulans. Bei der Intoxikation kommt es zu einer Euphorie mit gesteigerter Vitalität, übersteigerter Selbsteinschätzung hinsichtlich der Leistungsfähigkeit, Kreativität und Intelligenz, Beeinträchtigung des Urteilsvermögens, Geselligkeit oder Streitlust, Unruhe, Hypervigilanz, Hyperaktivität, sozialer und sexueller Enthemmung und vermindertem Schlafbedürfnis. Begleitet werden diese Symptome von psychomotorischer Erregung, Schwitzen, Mydriasis, Tachykardie, Hypertonie und einer Erhöhung der Atemfrequenz und Atemtiefe. Weiterhin kann es zu einem ausgeprägten Gefühl der Angst mit stereotypen Verhaltensweisen, akustischen, optischen bzw. taktilen Halluzinationen 
kommen sowie paranoiden Wahnvorstellungen mit dem Auftreten einer drogeninduzierten Psychose. Nach Abklingen der stimulierenden Rauschwirkung kommt es regelmäßig zu einem „depressiven Rauschstadium“ mit dysphorischer Verstimmung, die nicht selten von suizidalen Gedanken begleitet ist [13].

Cave: Suizidgedanken kommen nach der stimulierenden Rauschwirkung häufig vor.

Entzug. Der Kokainentzug ist durch ein charakteristisches Entzugssyndrom gekennzeichnet, dass sich innerhalb weniger Stunden bis Tage nach Beendigung des Konsums einstellt:

- Dysphorie

- Müdigkeit

- lebhafte, unangenehme Träume

- Insomnie oder Hypersomnie

- psychomotorische Hemmung oder Erregtheit

- vermehrter Appetit

- Lustlosigkeit und ein starkes Verlangen („Craving“) nach der Droge kommen häufig hinzu.

Das Entzugssyndrom verläuft in 3 Phasen [14]:

- Die 1. akute Phase („Crash“) mit starkem Drogenverlangen. Diese wird nach Perioden wiederholten und hoch dosierten Kokaingebrauchs („Runs“, „Binges“) beobachtet.

- In der 2. Phase des Entzugs folgen intensive, als unangenehm erlebte Gefühle, Müdigkeit, Mattigkeit, oft depressive Verstimmung bis hin zu Suizidalität, lebhafte unangenehme Träume, Schlaflosigkeit oder erhöhtes Schlafbedürfnis, Appetit, psychomotorische Getriebenheit oder Drosselung. Folgen sind ernsthafte Einschränkungen des psychosozialen Funktionsniveaus.

- Die 3. Phase erstreckt sich über einen längeren Zeitraum von bis zu 10 Wochen. Hier bilden sich die oben aufgeführten Entzugssymptome sukzessive zurück. Depressive Symptome und Suizidideen bis hin zu Suizidalität können jedoch deutlich darüber hinaus anhalten.

\section{Tipp für die Praxis}

Suizidale Gedanken und konkrete Absichten können in allen 3 Phasen des Entzugssyndroms auftreten und stellen eine Behandlungsindikation dar. Insbesondere bei starken Konsumenten von Kokain können kokaininduzierte Psychosen von tage- bis (selten) wochenlanger Dauer auftreten.
Somatische Komplikationen können während des Entzugs ebenfalls auftreten.

Aus neurologischer Sicht kann es gemäß der Leitlinie der Arbeitsgemeinschaft der wissenschaftlichen medizinischen Fachgesellschaften (AWMF) bei hohen Einnahmedosen zu einer zentralnervösen Übererregung mit Koordinationsstörungen, Verwirrtheit, Unruhe und zerebralen Krampfanfällen kommen [5]. Ferner können Dyskinesien und Dystonien sowie in besonders schweren Fällen Hyperpyrexien und Koma auftreten. Kardiovaskulär können kardiale Arrhythmien, Atemdepression und Brustschmerzen mit Dyspnoe bei Kokainrauchern entstehen [15]. Typische weitere Komplikationen sind Reizungen und Blutungen der Nasenschleimhäute, Sinusitiden sowie nekrotisierende Defekte im nasopharyngealen Bereich. Ein gehäuftes Auftreten von Infektionen wird insbesondere beim intravenösen Konsum beschrieben. In der Schwangerschaft können als Folge intrauteriner Kokainexposition ein retardiertes Wachstum des Fötus und ein erniedrigtes Geburtsgewicht, eine verminderte Plazentadurchblutung und fetale Hypoxie mit Spontanaborten auftreten [2].

\section{Tipp für die Praxis}

Aufgrund vasokonstriktorischer Effekte von Kokain besteht bei einer Kokain-Intoxikation immer das Risiko für das Auftreten von apoplektischen Insulten, Myokardinfarkten, kardialen Arrhythmien sowie Symptomen einer adrenergen Überfunktion [2].

Komorbidität. Untersuchungen in den USA weisen auf eine hohe Prävalenz von Kokaingebrauch bei schizophrenen Menschen hin. Häufiger ist die Kokainabhängigkeit mit einer affektiven Störung assoziiert. An Persönlichkeitsstörungen kommen komorbid häufig Borderline-, antisoziale, narzisstische, histrionische und paranoide Persönlichkeitsstörungen vor. Weiterhin ist die Kokainabhängigkeit häufig mit der Diagnose einer posttraumatischen Belastungsstörung und dem pathologischen Glücksspiel assoziiert [1, 16].

Therapie. Therapeutisch sollten zur Akuttherapie einer Kokain-Intoxikation bei gleichzeitig bestehender schwerer Angst und Erregung Benzodiazepine zum Einsatz kommen. Bei psychotischer Symptomatik sind kurzfristig Butyrophenone (z.B. Haloperidol), mittelfristig jedoch Neuroleptika der 2. Generation zu empfehlen. Starke vegetative Symptome sollten ebenfalls symptomatisch behandelt werden. Bei stationärer Auf- 
nahme für eine Entgiftung ist, bei der Gefahr von Blutdruckabfällen, Herz-Kreislauf-Stillstand und Koma, ein Monitoring der Vitalfunktionen durchzuführen.

Bei starkem Entzugssyndrom kann eine stationäre Aufnahme notwendig werden.

Eine Reihe von Studien belegt, dass beim Kokainentzugssyndrom am ehesten antriebssteigernde trizyklische Antidepressiva vom Typ Desipramin empfohlen werden können [2]. Zentrale Bestandteile der Behandlung der Abhängigkeit sind v.a. verhaltenstherapeutische und kognitive Therapien, die auf die Vermittlung alternativer Reaktionsmuster und einen besseren Umgang mit Gefährdungssituationen abzielen. Auch Rückfallvermeidungsverfahren und supportive Therapien werden eingesetzt.

Pharmakologisch sind zahlreiche Substanzen als Rückfallprophylaxe eingesetzt worden, darunter Antidepressiva (Desipramin, Imipramin, Fluoxetin), sog. Mood Stabilizer und Dopaminagonisten [1, 2].

\section{Amphetamin, Methamphetamin und Ecstasy}

\section{- Amphetamin oder Metamphetamin}

Diese beiden Substanzen sind wie die körpereigenen Neurotransmitter Dopamin, Noradrenalin und Adrenalin Derivate des Phenethylamins (Abb.2). Sie sind aufgrund ihrer Halbwertszeit (10 - 30 Stunden) länger wirksam als Kokain und werden daher in größeren Abständen konsumiert. Pharmakologisch werden sie als indirekt wirkende Katecholaminagonisten eingestuft, ihre zentrale und periphere Wirkung beruht auf Freisetzung von Dopamin und Noradrenalin aus den präsynaptischen Nervenendigungen. Weiterhin hemmt Amphetamin in hohen Dosen die Monoaminoxidase $[17,18]$.

Speed und Crystal sind häufige gängige Bezeichnungen in der Drogenszene für Amphetamin- und Methamphetamin. Als „Ice“ bzw. „Crack“ wird die Base von Methamphetamin bezeichnet. Der Konsum kann oral, inhalativ und intravenös erfolgen.

\section{Ecstasy}

Zu der Ecstasy-Gruppe gehören als bekannteste Vertreter:

- 3,4-Methylendioxy-N-methamphetamin (MDMA)

- die Analoga 3,4-Methylendioxy-N-ethamphetamin (MDE), 3,4-Methylendioxy-N-amphetamin (MDA)

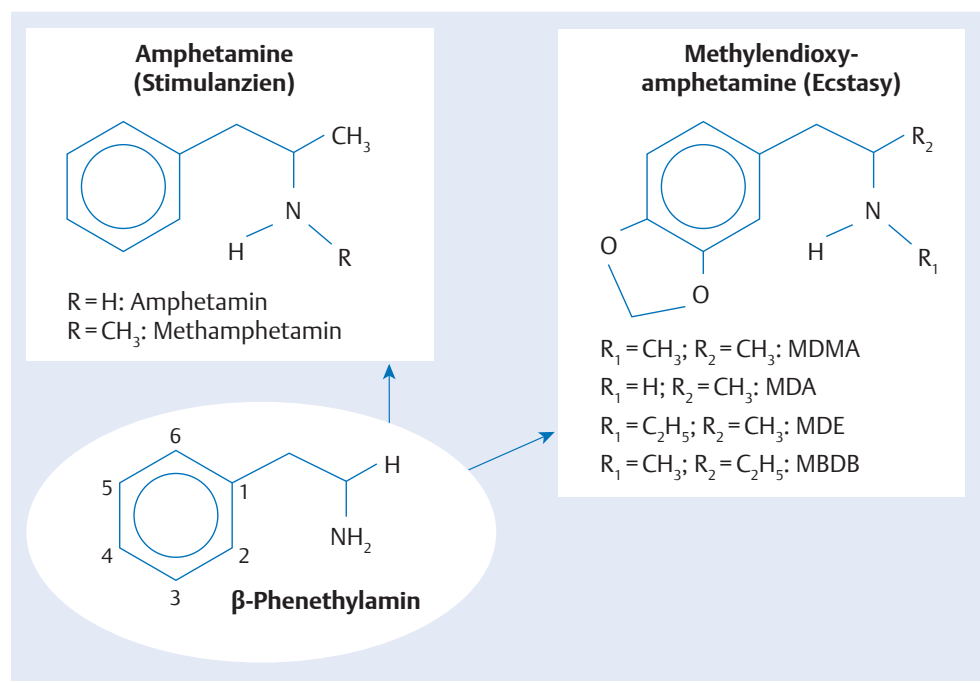

Abb. 2 Chemische Strukturformeln von Amphetaminstimulanzien und Ecstasy (Methylendioxyamphetamine).

und N-Methyl-1-1,3-benzodioxol-5-yl-2butanamin (MBDB)

Als wichtigste pharmakologische Wirkung führt Ecstasy zu einer verstärkten Ausschüttung und Hemmung der Wiederaufnahme von Serotonin und Dopamin aus dem synaptischen Spalt [19]. Der Konsum ist eng mit der Tanzszene verknüpft und wird dort fast immer in Tablettenform oral konsumiert. Die illegal konsumierten Tabletten enthalten durchschnittlich 60-120 mg MDMA oder MDMA-Analoga.

Klinische Symptome. Die Symptomatik bei einer Intoxikation mit Psychostimulanzien vom Amphetamintyp ist klinisch nicht von einer Kokain-Intoxikation zu unterscheiden. Vordergründig kommt es zu einem euphorischen „High“-Gefühl, subjektiv gesteigerter geistiger und körperlicher Leistungsfähigkeit, erhöhter Vigilanz und Unterdrückung von Schlaf, Müdigkeit sowie des Hungergefühls.

Die psychischen Akutwirkungen werden von körperlichen und vegetativen Symptomen begleitet: Tachykardie, Mydriasis, erhöhter Blutdruck, Schwitzen und psychomotorische Unruhe, gelegentlich auch Übelkeit, Erbrechen und thorakale Schmerzen. Als Komplikationen können Halluzination oder Wahn auftreten.

Bei kontinuierlichem Konsum von Amphetaminen/ Metamphetaminen entsteht eine rasche körperliche und pharmakodynamische Toleranz gegenüber den euphorisierenden und blutdrucksteigernden Wirkungen. Dabei kann es sogar zur Kollapsneigung bei körperlicher Anstrengung kommen. 
Entzug. Das Entzugssyndrom ist durch einen ausgeprägten Suchtdruck („Craving“) und Rebound-Phänomene wie Abgeschlagenheit, Schläfrigkeit, depressive Verstimmung, Ängste und Konzentrationsstörungen gekennzeichnet.

Ein vegetatives Entzugssyndrom im engeren Sinne ist nicht beschrieben.

Suizidalität kann auch hier eine Rolle spielen und gehört abgeklärt. Innerhalb einer Woche bildet sich das Entzugssyndrom in der Regel zurück. Als Komplikation treten bei chronischem Amphetamingebrauch aggressives Verhalten, Stereotypien und induzierte Psychosen auf. Insbesondere ein Beeinträchtigungs- und Verfolgungswahn („Speed Paranoia“) kann auftreten.

Induzierte Psychosen bei Amphetaminentzug

Bei den induzierten Psychosen sind optische und taktile Mikrohalluzinationen (Ameisenlaufen, Wanzen unter der Haut) sowie ein Alternieren zwischen ekstatischer Gehobenheit und ängstlich-depressivem Syndrom charakteristisch.

Die psychotischen Symptome entwickeln sich innerhalb von 48 Stunden nach der letzten Einnahme und klingen unter Abstinenz und vorübergehender Gabe von Neuroleptika in der Regel innerhalb von 7-10 Tagen, manchmal jedoch erst nach Wochen bis Monaten ab $[15,16,19]$. Nach Abklingen einer Ecstasy-Intoxikation geben Konsumenten häufig Erschöpfung, Kopfschmerzen, Ängstlichkeit und traurige Verstimmung an. Über die Intoxikation hinaus werden auch bei Ecstasy-Konsum Psychosen beobachtet, wobei es aufgrund eines polyvalenten Konsummusters dieser Konsumenten schwierig ist, diese Komplikation allein auf Ecstasy zurückzuführen. Gelegentlich werden auch Flashbacks oder Echophänomene beschrieben [19].

Somatische Komplikationen

Zu den Komplikationen eines hoch dosierten Amphetaminkonsums gehören:

- Herzrhythmusstörungen

- hypertensive Krisen mit Gehirnblutungen

- epileptische Anfälle

- Dyskinesien und Dystonie

- Bewusstseinstrübung bis zum Koma

Differenzialdiagnose. Differenzialdiagnostisch ist in erster Linie eine Kokain-Intoxikation oder eine andere
Intoxikation mit psychotropen Substanzen auszuschließen sowie eine Manie. In manchen Fällen ist differenzialdiagnostisch auch an Migräne, einen Schlaganfall, Myokardischämie oder neurologische Erkrankungen zu denken. Die Diagnose ist zum einen zu stellen durch die Anamnese, zum anderen durch den körperlichen Befund (evtl. Einstichstellen bei i.v. Konsum) sowie durch die entsprechenden Nachweise in Blut und Urin [2].

\section{Tipp für die Praxis}

Kokain und Amphetamine haben eine biphasische Wirkung. Zunächst manifestiert sich ein euphorisches Stadium, gefolgt von einem dysphorischen Stadium, welches unmittelbar in ein Entzugssyndrom übergeht. Entsprechend wird das dysphorische Stadium in der Regel durch erneuten Konsum zu bekämpfen versucht.

Therapie. Im Falle einer Intoxikation erfolgt die Therapie symptomatisch. Agitiertheit und maniforme Erregung können mit Sedativa behandelt werden. Haloperidol kann bei starker psychomotorischer Erregung und psychotischer Symptomatik eingesetzt werden. Bei im Vordergrund stehender Angst sollten Anxiolytika eingesetzt werden.

Die Wirksamkeit trizyklischer Antidepressiva beim Stimulanzienentzugssyndrom ist für Kokain gut belegt und dürfte bei Amphetaminen und Ecstasy ähnlich sein. Der Erfolg von dopaminerg wirksamen Substanzen (Lisurid, Amineptin) konnte bisher nicht überzeugend nachgewiesen werden. Bei psychotischen Rauschverläufen und induzierten psychotischen Störungen können vorübergehend Benzodiazepine und mit Vorsicht Neuroleptika symptomatisch eingesetzt werden. Bei zugrunde liegendem Konsum von Ecstasy sollten Neuroleptika jedoch möglichst vermieden werden, da sie analog zu den Erfahrungen bei halluzinogen induzierten psychotischen Rauschverläufen das Zustandsbild sogar verschlechtern können. Antidepressiva, besonders Serotonin-Wiederaufnahmehemmer, sind während der Intoxikation kontraindiziert, da sie zu der Entwicklung eines gefährlichen Serotoninsyndroms beitragen könnten [2].

\section{Antidepressiva sind aufgrund des Risikos eines Serotoninsyndroms kontraindiziert.}

Bei starken Nacheffekten kann es gelegentlich sinnvoll sein, Benzodiazepine streng zeitlich limitiert einzusetzen. Die Studienlage bzgl. einer Postakutbehandlung ist 
weiterhin dürftig und basiert auf Expertenmeinungen. Es wurden bisher vereinzelt Substitutionsversuche mit Amphetaminen oder Kalziumkanalblockern als AntiCraving-Substanzen getestet und es wurden (Teil-) Erfolge berichtet, die jedoch nur als vorläufig zu werten sind.

Hinsichtlich der Postakutbehandlung bei Ecstasy gibt es keine Evidenz zur Wirksamkeit einer pharmakotherapeutischen Behandlung [2]. Neben einer medikamentösen Behandlung sollten folgende Faktoren im Vordergrund zur Erhaltung der Abstinenz stehen:

- sozialpsychiatrische Maßnahmen

- ambulante Psychotherapien

- das Erreichen einer ausreichenden Tagesstruktur

- das Streben nach einer stabilen sozialen Situation

\section{Halluzinogene}

Von den unzähligen bekannten halluzinogenen Substanzen kann hier nur eine Auswahl der wichtigsten besprochen werden. Halluzinogene können eingeteilt werden in

- typische/klassische Halluzinogene und

- atypische Halluzinogene [2].

Klassische Halluzinogene. Zu den klassischen Halluzinogenen werden gezählt:

- Meskalin

- LSD

- Dimethyltryptamin (DMT)

- Psilocybin (Abb.3)

Es besteht eine chemische Verwandtschaft insbesondere zu Serotonin. Klassische Halluzinogene sind Indolderivate (DMT, LSD, Psilocybin) und Phenylethylaminderivate (z. B. Meskalin).

Atypische Halluzinogene. Diese unterscheiden sich von den klassischen Halluzinogenen durch eine zusätzlich sedierende Komponente. Als bekannte Beispiele sind Nachtschattengewächse wie Engelstrompete und Stechapfel zu nennen (sog. biogene Drogen) aber auch der Fliegenpilz und synthetische Substanzen wie Phenzyklidin (PCP) und Ketamin. Diese Substanzen beeinflussen die Aktivität von GABA- und Glutamat(NMDA-)Rezeptoren.

Ein physisches Abhängigkeitspotenzial ist nach heutigem Wissenstand für Halluzinogene nicht beschrieben.

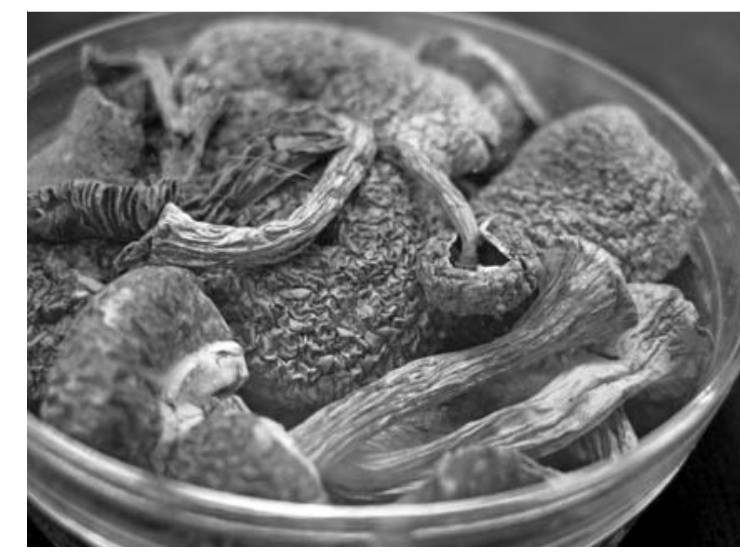

Abb. 3 Das Indolalkaloid Psilocybin kommt in einigen Pilzarten vor - insbesondere in der Gattung Psilocybe. Das Foto zeigt getrocknete „Magic Mushrooms“, deren Wirkung der des LSD ähnelt (Quelle: Thieme Verlagsgruppe).

\section{Intoxikation, Entzugssyndrom, neuropsychiatrische}

Störungen. Qualitativ sind die Effekte der verschiedenen klassischen Halluzinogene prinzipiell ähnlich, sie können jedoch interindividuell und sogar intraindividuell von Mal zu Mal sehr unterschiedlich ausfallen. Es kommt zu einem qualitativ veränderten Bewusstseinszustand, der oft als Bewusstseinserweiterung bezeichnet wird. Quantitative Einschränkungen der Bewusstseinslage, wie z.B. Vigilanzminderung und Eintrübung, fehlen in der Regel. Das Zeiterleben ist deutlich verändert, Umwelt und die eigene Person werde auf eine traumartige Weise erlebt mit Verschmelzungserlebnissen. Die Affektivität ist stark verändert, wobei Glücksgefühle, aber auch Traurigkeit und Angst erlebt werden können. Optische Wahrnehmungsveränderungen und Halluzinationen sind typische Symptome.

\section{Tipp für die Praxis}

Atypische Halluzinogene unterscheiden sich von den klassischen dadurch, dass sie selbst bei üblichen Dosierungen neben den qualitativen Bewusstseinsveränderungen auch dämpfende und sedierende Effekte bzw. eine Minderung der Vigilanz bewirken $[2,19]$.

Bei regelmäßigem Konsum kann es zu drogeninduzierten Psychosen von mehrwöchiger Dauer kommen. Die psychotische Symptomatik muss bei einer Drogenpsychose innerhalb von $48 \mathrm{~h}$ nach dem letzten Konsum aufgetreten sein, damit diese Diagnose gestellt werden kann [2]. Da der Anteil von affektiven Symptomen und schizophren-psychotischen Symptomen beiderseits hoch sein kann, ist das klinische Bild in der Regel auch 
einer Schizophrenie oder einer schizoaffektiven Störung ähnlich.

Dauert die psychotische Symptomatik länger als 6 Monate an, ist die Diagnose einer komorbiden Psychose aus dem schizophrenen Formenkreis zu stellen.

Eine weitere Komplikation sind die sog. Flashbacks (Echopsychosen) bzw. persistierende Wahrnehmungsstörungen, bei denen nach einem freien Intervall von Wochen bis Monaten nach dem letzten Rauscherleben die psychischen Phänomene des Rausches ohne erneute Substanzeinnahme für Sekunden bis Minuten, in seltenen Fällen auch andauernd auftreten [20].

Mydriasis, Tachykardie, Schwitzen, Palpitationen, verschwommenes Sehvermögen, Tremor, Anstieg der Körpertemperatur sowie Koordinationsstörungen können ebenfalls auf eine Intoxikation hinweisen. Schwerwiegende somatische Komplikationen sind bei dem Konsum klassischer Halluzinogene weniger zu befürchten [2].

\section{Tipp für die Praxis}

Überdosierungen von atypischen Halluzinogenen können zu einer ausgeprägten Bewusstseinstrübung, Orientierungsstörungen und deliranten Symptomen und zu potenziell lebensbedrohlichen intensivpflichtigen vegetativen Begleiterscheinungen führen (Atemlähmung, Koma) [19, 20].

Therapie. Neben „Talking Down“ kann man im Rahmen einer Intoxikation symptomatisch mit Benzodiazepinen behandeln. Eine antipsychotische Medikation ist bei einer Intoxikation durch Halluzinogene nicht indiziert, da Antipsychotika verstärkend auf Gereiztheit und aversiv empfundene Emotionen wirken. Es besteht zudem derzeit kein Hinweis auf eine Wirksamkeit dieser Stoffgruppe auf die Symptomatik. Bei Flashbacks können Benzodiazepine eingesetzt werden oder ein Behandlungsversuch mit Serotonin- und NoradrenalinWiederaufnahmehemmern, Clonidin oder Naltrexon durchgeführt werden [19]. Bei protrahiert verlaufenden konsuminduzierten Psychosen sollte ein Behandlungsversuch mit Lithium und vorübergehend Benzodiazepinen geprüft werden. Ebenfalls gibt es Hinweise auf die Wirksamkeit von Elektrokrampftherapie bei therapieresistenten psychotischen Störungen $[19,20]$.

\section{Kernaussagen}

- Der Konsum illegaler Drogen und die Entstehung von Suchterkrankungen können sowohl durch genetische als auch Umweltfaktoren bedingt sein.

- Ein schädlicher Gebrauch einer Substanz liegt vor, wenn infolge des Substanzkonsums bereits eine gesundheitliche Schädigung körperlicher oder psychischer Art eingetreten ist.

- „Partydrogen“ (Kokain, Amphetamine, Halluzinogene) haben eine stimulierende Wirkung und werden häufig gegenseitig austauschbar konsumiert.

- Unbehandelt verlaufen Suchterkrankungen häufig chronisch progredient.

- Suizidalität kann bei Abhängigkeitserkrankungen eine Rolle spielen und sollte grundsätzlich abgeklärt werden.

\section{Über die Autoren}

\section{Alexander Glahn}

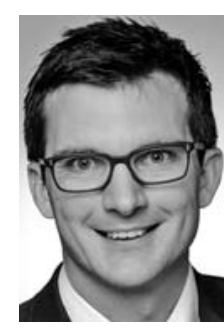

Dr. med. Jahrgang 1978. Studium der Sportwissenschaft und Anglistik (Lehramt für Gymnasien) und Humanmedizin in Hannover und Facharztweiterbildung Psychiatrie und Psychotherapie (Klinik für Psychiatrie, Sozialpsychiatrie und Psychotherapie der Medizinischen Hochschule Hannover). Zusatzbezeichnung Suchtmedizinische Grundversorgung. Qualifikation und Zertifizierung als Kursleiter für das vom IFT angebotene „Rauchfrei-Programm“. Funktionsoberärztliche Tätigkeit in der Klinik für Psychiatrie, Sozialpsychiatrie und Psychotherapie (Med. Hochschule Hannover).

\section{Thomas Hillemacher}

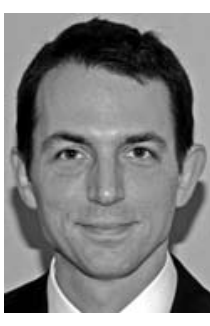

Prof. Dr. med. Jahrgang 1972. 1993 2000 Studium der Medizin in Erlangen und Córdoba/Spanien. 2002 Promotion, 2006 Habilitation mit dem Thema „Neurobiologische Mechanismen des Craving bei Patienten mit Alkoholabhängigkeit“. 2006 Facharzt für Psychiatrie und Psychotherapie, Fachkunde suchtmedizinische Grundversorgung, Zusatzbezeichnung Geriatrie. Seit 2008 leitender Oberarzt und stellvertretender Klinikdirektor an der Klinik für Psychiatrie, Sozialpsychiatrie und Psychotherapie an der Medizinischen Hochschule Hannover. 


\section{Stefan Bleich}

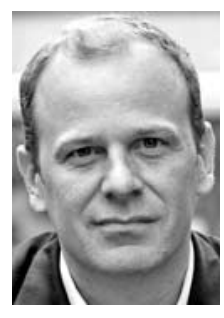

Prof. Dr. med. Jahrgang 1968. Facharzt für Psychiatrie und Psychotherapie, Zusatzbezeichnung Klinische Geriatrie und Schwerpunktbezeichnung Forensische Psychiatrie. Seit 2008 Direktor der Klinik für Psychiatrie, Sozialpsychiatrie und Psychotherapie der Medizinischen Hochschule Hannover. Forschungsschwerpunkte: Substanzabhängigkeiten, Psychopharmakologie und Analysen zur epigenetischen Regulation. Seit 2011 Präsident der AMSP und seit 2013 1. Vorsitzender der AGNP.

Interessenkonflikt: T. Hillemacher ist Mitglied in einem Beratergremium für Lundbeck und erhielt Honorare und Zuwendungen von den Firmen Servier, Otsuka, Lundbeck, Bristol-Myers Squibb, Desitin und D\&A Pharma. Zudem war er für die Arbeitsgemeinschaft für Neuropsychopharmakologie und Pharmakopsychiatrie (AGNP) an der Leitlinienentwicklung tabak- und alkoholbezogene Störungen beteiligt.

S. Bleich erhielt Honorare und Zuwendungen von Lundbeck und Servier. Zudem ist er Vorsitzender der Arbeitsgemeinschaft für Neuropsychopharmakologie und Pharmakopsychiatrie (AGNP) sowie des Instituts für Arzneimittelsicherheit in der Psychiatrie AMSP e. V.

Bei A. Glahn bestehen keinerlei Interessenkonflikte.

\section{Korrespondenzadresse}

Dr. med. Alexander Glahn

Klinik für Psychiatrie, Sozialpsychiatrie und

Psychotherapie, Medizinische Hochschule Hannover

Carl-Neuberg-Straße 1

30625 Hannover

E-Mail: glahn.alexander@mh-hannover.de

Verantwortlicher Herausgeber für diesen Beitrag:

Prof. Dr. med. Fritz Hohagen, Lübeck

\section{Literatur}

1 Batra A. Praxisbuch Sucht: Therapie der Suchterkrankungen im Jugend- und Erwachsenenalter. Stuttgart, New York: Thieme; 2012

2 Müller H, Laux G, Deister A et al. Psychiatrie, Psychosomatik und Psychotherapie. Berlin: Springer; 2013

3 Dilling $\mathrm{H}$, Freyberger $\mathrm{HJ}$. World Health Organization. Taschenführer zur ICD-10-Klassifikation psychischer Störungen mit Glossar und diagnostischen Kriterien sowie Referenztabellen ICD-10 vs. ICD-9 und ICD-10 vs. DSM-IV-TR. 7. überarb Aufl. unter Berücks. der Änderungen entspreched ICD-10GM (German Modification) 2014 ed. Bern: Huber; 2014

4 Falkai P, Wittchen H, Döpfner M. American Psychiatric Association. Diagnostisches und statistisches Manual psychischer Störungen DSM-5. Göttingen: Hogrefe; 2015

5 Thomasius R, Gouzoulis-Mayfrank E, Wiedenmann $\mathrm{H}$ et al. AWMF-Behandlungsleitlinie: Psychische und Verhaltensstörungen durch Kokain, Amphetamine, Ecstasy und Halluzinogene. Fortschr Neurologie Psychiatrie 2004; 72: 679-695

6 Simon R, Spegel H, Hüllinghorst R et al. Bericht des Nationalen REITOX Knotenpunkts für Deutschland an die EBDD: Drogensituation. München: DBDD; 2001

7 Pfeiffer-Gerschel T. European Monitoring Centre for Drugs and Drug Addiction. European Monitoring Centre for Drugs and Drug Addiction. Drogensituation. Bericht 2013 des nationalen REITOX-Knotenpunkts an die EBDD; neue Entwicklungen und Trends; Deutschland. München: European Monitoring Centre for Drugs and Drug Addiction, Dt. Beobachtungsstelle; 2014

8 Wittchen $\mathrm{H}$. World Health Organization. Composite International Diagnostic Interview (CIDI) Interviewerheft. Weinheim: Beltz Test; 1990

9 Wittchen $\mathrm{H}$. World Health Organization. Eine Einführung und Durchführungsbeschreibung zum Composite International Diagnostic (CIDI) Manual. Weinheim: Beltz Test; 1990

10 Miller WR, Rollnick S, Brueck R. Motivierende Gesprächsführung. 3.: Aufl. Freiburg im Breisgau: Lambertus; 2009

11 Osterloh J. Testing for drugs of abuse. Pharmacokinetic considerations for cocaine in urine. Clin Pharmacokinet 1993; 24 $355-361$

12 Holsboer F. Handbuch der Psychopharmakotherapie mit 155 Tabellen. Berlin, Heidelberg: Springer; 2008

13 Zerdick J. Suchtmedizinischer Kongress. Entwicklungen in der Suchtmedizin. 7. Suchtmedizinischer Kongress der DGDS, 6.8. November 1998 in Berlin. Berlin: VWB, Verl. für Wiss. und Bildung; 1999

14 Gawin FH, Kleber HD. Abstinence symptomatology and psychiatric diagnosis in cocaine abusers. Clinical observations. Arch Gen Psychiatry 1986; 43: 107 - 113

15 Gold MS, Graham NA, Kobeissy FH et al. Speed, cocaine, and other psychostimulants death rates. Am J Cardiol 2007; 100: 1184

16 Gouzoulis-Mayfrank E, Scherbaum N. Drogenabhängigkeit (ICD-10 F1). In: Vorderholzer U, Hohagen F, Hrsg. Therapie psychischer Erkrankungen, State of the art. 7. Aufl. München, Jena: Urban \& Fischer; 2013: 35 -48

17 Lee TH, Ellinwood EHJr, King GR. Regional differences in the effects of amphetamine withdrawal on dopamine dynamics in the striatum. Neuropsychopharmacol 1997; 16: $311-314$

18 Silvia CP, Jaber M, King GR et al. Cocaine and amphetamine elicit differential effects in rats with a unilateral injection of dopamine transporter antisense oligodeoxynucleotides. Neurosci 1997; 76: $737-747$

19 Gouzoulis-Mayfrank E, Bilke O. Psychostimulanzien. In: Batra A, Bilke O, Hrsg. Praxisbuch Sucht. Stuttgart: Thieme Verlag; 2011: 181 - 187

20 Abraham HD, Aldridge AM, Gogia P. The psychopharmacology of hallucinogens. Neuropsychopharmacol 1996; 14: $285-298$ 


\section{CME。thieme.de}

\section{CME-Fragen}

CME-Teilnahme

- Viel Erfolg bei lhrer CME-Teilnahme unter http://cme.thieme.de

- Diese Fortbildungseinheit ist 12 Monate online für eine CME-Teilnahme verfügbar.

- Sollten Sie Fragen zur Online-Teilnahme haben, unter http://cme.thieme.de/hilfe finden Sie eine ausführliche Anleitung.

1

Was gehört nicht zu den ICD-10Kriterien der Abhängigkeitserkrankung?
A starkes, oft unüberwindbares Verlangen, die Substanz einzunehmen

B Schwierigkeiten, die Einnahme zu kontrollieren (was den Beginn, die Beendigung und die Menge des Konsums betrifft)

C körperliche Entzugssymptome

D Benötigen immer größerer Mengen, damit die gewünschte Wirkung eintritt

E Zur Diagnose eines Abhängigkeitssyndroms müssen nach der ICD-10 mindestens 3 der dort genannten Kriterien während der letzten 6 Monate gemeinsam erfüllt gewesen sein.
Der Kokainentzug ist durch ein charakteristisches Entzugssyndrom gekennzeichnet. Was trifft nicht zu?

3

Welche Aussage ist richtig?

4

Was gehört nicht zu den typischen Komplikationen eines hoch dosierten Amphetaminkonsums?
A Herzrhythmusstörungen
B hypertensive Krisen mit Gehirnblutungen
C Miosis
D epileptische Anfälle
E Dyskinesien und Dystonie

Was wird nicht den klassischen Halluzinogenen zugeordnet?
A Phenzyklidin (PCP)
B Meskalin
C Lysergsäurediethylamid (LSD)
D Dimethyltryptamin (DMT)
E Psilocybin 


\section{CME-Fragen}

Psychische und Verhaltensstörung durch Kokain, Amphetamine, Ecstasy und Halluzinogene

\section{6}

Welche Aussage ist richtig?

\section{7 \\ 7}

Welche Aussage trifft nicht zu?
A Atypische Halluzinogene unterscheiden sich von den klassischen dadurch, dass sie selbst bei üblichen Dosierungen neben den qualitativen Bewusstseinsveränderungen auch dämpfende und sedierende Effekte bzw. eine Minderung der Vigilanz hervorrufen.

B Eine antipsychotische Medikation ist bei einer Intoxikation durch Halluzinogene indiziert, da diese verstärkend auf Gereiztheit und aversiv empfundene Emotionen wirken.

C Die psychotische Symptomatik muss bei einer Drogenpsychose innerhalb von $24 \mathrm{~h}$ nach dem letzten Konsum aufgetreten sein, damit diese Diagnose gestellt werden kann.

D Halluzinogene haben eine biphasische Wirkung. Zunächst manifestiert sich ein euphorisches Stadium, gefolgt von einem dysphorischen Stadium, welches unmittelbar in ein Entzugssyndrom übergeht.

E Bekannte Beispiele für typische Halluzinogene sind Nachtschattengewächse wie Engelstrompete und Stechapfel.

Welche Aussagetiift nicht zu?

A Nach Abklingen einer Ecstasy-Intoxikation geben Konsumenten häufig Erschöpfung, Kopfschmerzen, Ängstlichkeit und traurige Verstimmung an.

B Über die Intoxikation hinaus werden auch bei Ecstasy-Konsum Psychosen beobachtet.

C Ein polyvalentes Konsummuster kommt bei Konsumenten von Ecstasy selten vor.

D Gelegentlich werden auch Flashbacks oder Echophänomene beschrieben.

E Kokain ist ein starkes Psychostimulans.

\section{8}

Welche Aussage ist richtig?
A Amphetamin oder Metamphetamin sind wie die körpereigenen Neurotransmitter Dopamin, Noradrenalin und Adrenalin Derivate des Phenethylamin.

B Amphetamin beschleunigt in hohen Dosen die Bildung der Monoaminoxidase.

C Speed und Crystal sind häufige gängige Bezeichnungen in der Drogenszene für Halluzinogene.

D „Ice“ bzw. „Crack“ sind Bezeichnungen für Kokain.

E Als wichtigste pharmakologische Wirkung führt Ecstasy zu einer verminderten Ausschüttung von Serotonin und Dopamin aus dem synaptischen Spalt.
Was ist keine komorbide Störung bei Kokainabhängigkeit?
A Schizophrenie
B affektive Störung
C emotional instabile Persönlichkeitsstörung vom Borderline-Typ
D narzisstische Persönlichkeitsstörungen
E hypochondrische Störung

\section{0}

Welche Aussage trifft nicht zu? Merkmale der motivierenden Gesprächsführung sind...
A empathische Grundhaltung mit Verzicht auf Konfrontation.
B geschlossene Fragestellung mit implizierter Bewertung.
C Förderung der Wahrnehmung von Diskrepanzen zwischen Selbstbild und Wirklichkeit.
D Aufbau von Selbstvertrauen bezüglich der Fähigkeit zur Abstinenz.
E Vereinbarung von gemeinsam erarbeiteten und umschriebenen Behandlungszielen. 